\title{
Diseño de redes de autobús frente a la dispersión de la movilidad urbana
}

\author{
Hugo Badia Rodriguez \\ Doctorando, Universidad Politécnica de Cataluña, España
}

\section{RESUMEN}

Este artículo busca dar respuesta a la discusión sobre qué tipo de estrategia de red es la más adecuada: (i) una basada en conexiones directas, u (ii) otra basada en transferencias. En el estudio también se ha introducido el análisis de una red puramente radial, origen de las otras dos. A través de un modelo analítico se comparan los costes totales que implica trabajar con cada uno de los esquemas de red analizados para diferentes escenarios de dispersión de la movilidad urbana. De ello se obtienen los rangos de aplicabilidad de dichas estructuras vinculados a ese grado de dispersión. El fenómeno de descentralización de las actividades ha sido creciente en los entornos urbanos, hecho que dificulta mantener conectada la ciudad a través de redes radiales, e incluso de viajes directos. Esa es una de las principales razones para justificar un cambio de estructura basada en transferencias.

A su vez, el artículo incluye la manera en la que los resultados del modelo analítico pueden ser extrapolados a ciudades reales. Es decir, conocido el grado de dispersión de la demanda en una ciudad, poder determinar que estructura de red es más adecuada. Para el análisis de la descentralización de la demanda se parte de la información que aportan matrices origendestino que permiten obtener índices de autocorrelación espacial y de desigualdad. Ambos índices se complementan para conocer la estructura de la ciudad y su grado de descentralización. En este caso, se trabaja con la ciudad de Barcelona, que presenta un grado de descentralización que justifica cambiar a una estructura en base a transferencias.

\section{INTRODUCCIÓN}

Este artículo busca dar respuesta a la discusión existente en el ámbito del diseño de redes de autobús sobre que estructura de red es óptima. Existen dos planteamientos básicos: (i) aquel en el que se defiende conectar de manera directa el mayor número posible de orígenes y destinos, y (ii) aquel que apuesta por una red simplificada donde la sinergia entre líneas a través de transferencias permita satisfacer la totalidad de los viajes.

El principal argumento de los defensores del primer planteamiento es la premisa de que los usuarios son adversos a realizar transferencias, hecho que los disuade del uso del transporte público. Es por ello que defienden que deben ser reducidas al máximo, como planteaba Zhao (2006) en su modelo de diseño de redes. Por el contrario, otros autores defienden el segundo planteamiento. Mees (2000) sostenía que una red basada en transferencias es más eficiente 
y efectiva por su mayor legibilidad y fácil operativa.

Thompson and Matoff (2003) compararon diferentes redes de autobús, mostrando que aquellas diseñadas bajo el segundo enfoque presentaban mayores niveles de demanda. Relacionaban su mayor rendimiento a su mejor adaptación a las nuevas necesidades de desplazamiento resultado del proceso de descentralización de actividades que padecen los entornos urbanos. Anas et al (1998) expone la evolución de la ciudad en los dos últimos siglos. Partiendo de un escenario de alta centralización con un fuerte centro, la ciudad ha ido dispersándose progresivamente. Inicialmente con la ubicación de las actividades en el espacio adyacente a ese centro tradicional, y con el paso del tiempo, con la aparición de nuevos polos de actividades. Sin embargo, autores como Craig y Ng (2001), analizando la densidad de empleos, observaban que aunque nuevos espacios de actividades aparecían, el centro tradicional seguía presentando mayor relevancia, aunque este podía estar más o menos extendido.

En esta línea, Bertaud (2004) expone que la ciudad ha evolucionada hacia una estructura mono-policéntrica. Aunque aparecen nuevos polos atractores estos son subordinados respecto al centro. La ciudad no es la suma de distritos independientes, sino que sigue siendo un todo que tiende más bien a la dispersión.

La idoneidad de cada uno de los dos enfoques de red expuestos anteriormente se discutió en Thompson (1977) o Jara-Díaz y Gschwender (2003) en base a enfoques analíticos. Sin embargo, ambos estudios presentaban sus limitaciones. El primero comparaba una red mallada basada en transferencias con un sistema de servicios directos que conectaba las distintas zonas de la ciudad todas con todas. Esta última red no tenía en cuenta el grado de descentralización de la demanda a servir, no estando justificada la conexión de todos con todos los puntos de manera directa. Este hecho derivaba en una red muy costosa que difícilmente podía competir con una red mallada.

El estudio de Jara-Díaz y Gschwender (2003) en realidad no es el análisis de una red, es el de superponer dos corredores que se cruzan. Simplificación que deja al margen factores importantes de la comparativa de ambos enfoques. El coste de acceso, la longitud del trayecto o de infraestructura entre otros.

En el presente artículo se va más allá con el análisis de una red completa a la vez que se tiene en cuenta el grado de dispersión en que se encuentra la ciudad a estudiar. El objetivo último es dilucidar cuál de los dos enfoques es más adecuado en los distintos escenarios de dispersión de la movilidad. Basándose en modelos analíticos, tres estructuras de red son comparadas desde el punto de vista de los costes totales del sistema. La primera representan a una red completamente radial, estado inicial de las otras dos estructuras, las que representan los dos enfoques contrapuestos anteriormente. 
Por otro lado, se conectan los resultados del modelo analítico con el grado de descentralización de la demanda que presenta una ciudad real, en este caso la ciudad de Barcelona. De este modo, el planteamiento aquí presentado puede ser una herramienta adecuada para conocer en qué escenario se encuentran los entornos urbanos actualmente y que decisiones se deberían tomar a la hora de diseñar las redes de autobús.

El modelo analítico utilizado se presenta en la Sección 2. Sus resultados se recogen en la Sección 3. En la Sección 4 se expone el análisis del nivel de dispersión de la ciudad de Barcelona y su relación con los resultados analíticos previos. Finalmente, la sección 5 recoge las principales conclusiones del estudio.

\section{MODELIZACIÓN ANALÍTICA}

El modelo analítico utilizado se basa fundamentalmente en el modelo presentado en Daganzo (2010) y su posterior extensión en Estrada et al (2011). Sin embargo, algunas modificaciones respecto a la distribución de la demanda y la estructura de red son asumidas. La ciudad se esquematiza como un cuadrado de lado $D(\mathrm{~km})$, una demanda horaria media $\lambda$ ( $\mathrm{pax} / \mathrm{h}$ ) y una demanda en hora punta $\Lambda$ (pax/hp) deterministas.

Para poder tener en cuenta el fenómeno de la dispersión de la movilidad, el supuesto de que la demanda se distribuye uniformemente por toda la ciudad debe ser modificado. Pero como mostró Vaughan (1986), una distribución más próxima a la realidad complica en exceso la formulación, perdiendo la simplicidad y compacidad que caracteriza las formulaciones analíticas.

Por ello, la uniformidad se mantiene, pero no del mismo modo en toda la ciudad. Se distinguen dos áreas, una central y otra periférica. La demanda generada se distribuye uniformemente en ambas, pero con una densidad de viajes diferente. En cambio, la demanda es únicamente atraída en el área central, donde también se distribuye uniformemente. En todo caso, la demanda generada y atraída son independientes.

Para representar dicha distribución de demanda dos nuevos parámetros son introducidos: (i) el parámetro $\phi$ que marca el tamaño del área central, y adopta valores entre 0 y 1 , y (ii) el parámetro $f_{d}$ que indica cuanto mayor es la densidad de demanda generada en el área central respecto a la periferia. Este área central es un cuadrado de lado $\phi D$ cuya densidad de demanda generada es $f_{d}$ veces superior a la del resto de la ciudad. En el caso que el valor de $f_{d}$ valor sea igual a 1, la demanda generada se distribuye uniformemente por igual en toda la ciudad. En caso que su valor sea superior, el área central presenta una mayor capacidad generadora de demanda.

Debido a esta distribución de la demanda, existen dos tipos de viajes dependiendo de la localización de su origen, si este está localizado en el área central, o por contra, se sitúa en 
la periferia. La probabilidad de que sea un viaje central es $\rho$, y la de un viaje periferia-centro es el complementario 1- $\rho$. El valor de $\rho$ es dependiente de los dos anteriores parámetros, $\rho=$ $f_{d} \phi^{2} /\left[1+\phi^{2}\left(f_{d}-1\right)\right]$.

La Figura 1 muestra las dos estructuras de red que representan cada uno de los enfoques previamente expuestos. Un esquema híbrido como el presentado en Daganzo (2010), que representa una red basada en transferencias, y un esquema alternativo que conecta las distintas zonas del área central atractora con el resto de la ciudad directamente.

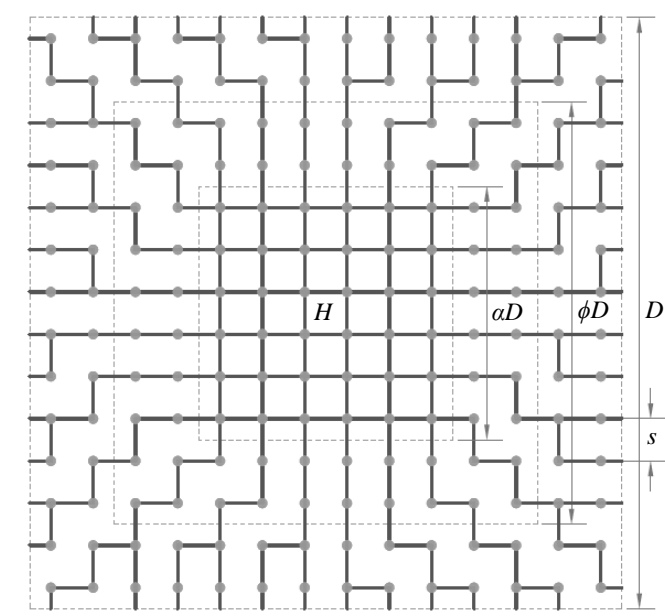

(a)Red híbrida basada en transferencias

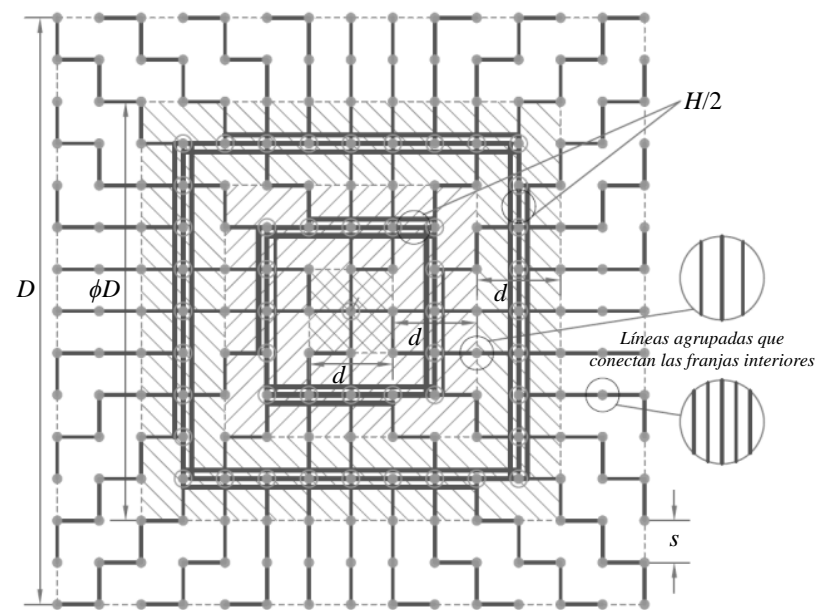

(b)Red basada en servicios directos

Fig. 1 - Estructuras de red comparadas

El esquema híbrido se compone de dos áreas, una malla central y una estructura ramificada en la periferia. Tres variables de decisión configuran la red: el tamaño de la malla viene definido por el parámetro $\alpha$, el intervalo de paso de sus corredores es $H$, y finalmente, la tercera variable es el espaciamiento entre paradas o líneas $s$, que marca la ramificación de las líneas en la periferia.

En el caso de la red de servicios directos, el primer paso es entender la zonificación del área central atractora. Como muestra las Figura 1b, este área se divide en franjas concéntricas de ancho $d$, una de las variables de decisión del modelo. La franja central es un cuadrado de lado $d$, y es servida por una red radial equivalente a un modelo híbrido para $\alpha=0$. De este modo, dicha franja está conectada con la totalidad de la ciudad. El intervalo conjunto de las líneas que atraviesan el centro en cada dirección es $H$, y el espaciamiento entre paradas $s$, completando la terna de variables que configuran la red. La siguiente franja ya está conectada con la franja anterior, y solo debe conectarse con el resto de la ciudad. Para ello nuevas líneas se superponen a las ya existentes.

Estas nuevas líneas se agrupan en un corredor central de la franja que sirven, y se ramifican sobre el territorio exterior siguiendo el mismo esquema que el de las líneas de la franja anterior. Cuatro grupos de líneas conectan toda la franja, distinguiéndose en función del cuadrante de ciudad donde se ramifican y el cuadrante de la franja donde se agrupan. Las 
líneas que se agrupan en el cuadrante Este de la franja se ramifican sobre los cuadrantes Norte y Sur de la ciudad, al igual que las que se agrupan en el cuadrante Oeste de la franja. Las que se agrupan en los cuadrantes Norte y Sur de la franja se ramifican por los cuadrantes Este y Oeste de la ciudad. El conjunto de líneas resultante se complementa de tal modo que conecta todos los cuadrantes de la franja con todos los cuadrantes de la ciudad. El intervalo de paso conjunto de cada uno de los grupos es $H$, resultando el intervalo final de las líneas que recorren la franja longitudinalmente $H / 2$. Esto es debido a que al grupo de líneas que se agrupan en un cuadrante de la franja se le superponen las líneas de sus cuadrantes adyacentes, ya que es sobre ese cuadrante donde se ramifican.

Generalizando, cada franja ya está conectada con las franjas interiores, y solo debe conectarse con el área exterior. Para ello, se vuelven a introducir nuevas líneas que recorren la franja a conectar y se ramifican para llegar a la totalidad de su área exterior. Se repite la misma disposición de líneas explicada en el párrafo anterior.

Por último, la red radial es el estado inicial de ambos esquemas. Es una red híbrida en la que la malla central no existe, es decir, la variable $\alpha$ es 0 , o es una red de conexiones directas donde solo existe la franja central.

\subsection{Función objetivo y formulación del modelo}

Para la optimización de la configuración de red se busca minimizar el coste total del sistema $Z$. Es por ello que la función objetivo del modelo (1) incluye costes parciales tanto del operador $C_{A}$ como del usuario $C_{U}$. Desde el punto de vista del operador se incluyen tres costes, los derivados de la longitud de red $L(\mathrm{~km})$, los kilómetros recorridos $V$ (veh- $\mathrm{km} / \mathrm{h}$ ) y la flota utilizada $M$ (veh-h/h) por hora de servicio. Estos costes son convertidos a unidades de tiempo y repercutidos por usuario, se multiplican por el factor $\epsilon_{i} / \mu \lambda$, coste unitario $\epsilon_{i}$ dividido por el valor del tiempo $\mu$ y la demanda horaria media $\lambda$.

Por otro lado, se incluyen los costes de las distintas etapas de la cadena de transporte del usuario: acceso $A(\mathrm{~h})$, espera $W(\mathrm{~h})$, trayecto $T(\mathrm{~h})$ y transferencia $\operatorname{Tr}(\mathrm{h})$. Estos costes son ponderados por la percepción que el usuario tiene de cada uno de ellos a través de los pesos $w_{i}$. El coste de transferencia $\operatorname{Tr}$ es el resultado de multiplicar el número medio de transferencias por viaje $e_{T}$ y el coste de una transferencia que se supone constante $\operatorname{tr}(\mathrm{h})$.

$$
\begin{gathered}
\min \left\{Z=C_{A}+C_{U}=\left[\epsilon_{L} L+\epsilon_{V} V+\epsilon_{M} M\right] / \lambda \mu+\left[w_{A} A+w_{W} W+w_{T} T+w_{T r} T r\right]\right\} \\
\text { sujeto a: } \\
\quad s>0 ; H>0 ; s / D \leq \min \{\alpha ; \phi\} ; O \leq C /(S F) \text {, red basada en transferencias } \\
s, d>0 ; H>0 ; s / D \leq \phi / 2 ; d / D \leq \phi / 2 ; O \leq C /(S F) \text {, red basada en servicios } \\
\text { directos }
\end{gathered}
$$

La configuración de red óptima está condicionada por las restricciones (1a) del valor de las variables de decisión y la ocupación del vehículo $O$ por limitaciones de su capacidad $C$. Esta 
capacidad está corregida por un factor de seguridad $S F$ por posibles picos de demanda dentro de la propia hora punta.

En las siguientes tablas se recogen las fórmulas de los diferentes costes parciales que componen la función objetivo para las dos estructuras de red estudiadas. En este artículo se presenta directamente la formulación para ambas estructuras. Una explicación más detallada puede encontrarse en Badia et al (2016). Otros parámetros de entrada del modelo que aparecen en la formulación son los vinculados a la circulación y los tiempos en parada: velocidad de circulación $v_{F F S}(\mathrm{~km} / \mathrm{h})$, tiempo por realizar una parada $\tau$ (s) y el tiempo de embarque por pasajero $\tau^{\prime}(\mathrm{s})$.

\begin{tabular}{cc}
\hline $\begin{array}{c}\text { Coste } \\
\text { parcial }\end{array}$ & Fórmula \\
\hline$L$ & $L=D^{2} / s$ \\
$V$ & $V=6 D / H$ \\
$M$ & $M=V / v_{c}$ \\
$v_{c}$ & $v_{c}=1 /\left[1 / v_{F F S}+\tau / s+\tau^{\prime}\left(1+e_{T}\right) \Lambda / V\right]$ \\
$A$ & $A=s / w$ \\
$W$ & $E=H\left[15 D\left(1+2 \phi+2 \phi^{2}\right)-15 s(1+\phi)-\rho(15 D+s(1+\phi))\right] / 45 s(1+\phi)$ \\
$E$ & $E=\left[15 D\left(1+2 \phi+2 \phi^{2}\right)-15 s(1+\phi)-\rho(15 D+s(1+\phi))\right] / 30(1+\phi)$ \\
$T$ & $T=E / v_{c}$ \\
$e T$ & $e_{T}=1-s(3+\rho) / 3 \phi D$ \\
$O$ & $O=(S F) H \Lambda\left[6 \phi D-s\left(3+\phi^{2}\right)\right] / 24 \phi D$ \\
\hline
\end{tabular}

\section{Tabla 1 - Formulación de los costes parciales de la estructura radial}

En el caso de la red basada en conexiones directas se permite trabajar con dos tipos de servicios diferentes, es decir, por intervalos de paso o por horarios. Se sigue el esquema utilizado en Tirachini et al (2010), donde si el intervalo de paso es menor que un determinado umbral $H_{s}$, se asume que el usuario trabaja por intervalos ya que desconoce el horario exacto de paso del autobús por parada. En este caso, el tiempo de espera se supone la mitad del intervalo de servicio. En caso contrario, el usuario tiene información sobre la hora de paso del autobús, adaptando su llegada a la parada a ese momento. El tiempo de espera es la suma de un tiempo de seguridad $h_{s}$, el usuario llega unos minutos antes para evitar perder el autobús por si este se adelanta, más un tiempo que penaliza el coste de oportunidad derivado de un nivel de servicio bajo, que depende del intervalo de paso. Ese coste de oportunidad es el resultado de multiplicar el intervalo de servicio por un factor $f_{s}$.

Para identificar que parte de la ciudad trabaja por horarios y cual por intervalos se define un nuevo parámetro $\varepsilon_{H}$. Este nos indica el tamaño del área central que trabaja por intervalos, un cuadrado de lado $\varepsilon_{H} D$. Fuera de esta área el servicio es operado por horarios. $\varepsilon_{H}$ es igual a la relación $H_{s} s / H D$, si esta es mayor que uno todo el sistema funciona por intervalos.

\begin{tabular}{ll}
\hline $\begin{array}{c}\text { Coste } \\
\text { parcial }\end{array}$ & Fórmula \\
\hline
\end{tabular}




\begin{tabular}{|c|c|}
\hline$L$ & $L=D^{2}\left(1+\alpha^{2}\right) / s$ \\
\hline$V$ & $V=2 \alpha D^{2}(3-\alpha) / s H$ \\
\hline$M$ & $M=V / v_{c}$ \\
\hline$v_{c}$ & $v_{c}=1 /\left[1 / v_{F F S}+\tau / s_{S}+\tau^{\prime}\left(1+e_{T}\right) \Lambda / V\right]$ \\
\hline \multirow[t]{2}{*}{$A$} & $A=s / w$ \\
\hline & If $\alpha>\phi$ \\
\hline$W$ & $W=H \frac{\phi^{2} D^{2}\left[\left(2+\alpha^{3}\right)(1-\rho)+3 \alpha(1+\rho)-6 \alpha \phi^{2}\right]-3 \phi D s\left[\phi\left(1+\alpha^{2}\right)(1-\rho)+2 \alpha\left(\rho-\phi^{2}\right)\right]+3 \alpha s^{2}\left(1-\phi^{2}\right) \rho}{6 \alpha \phi^{2}\left(1-\phi^{2}\right) D^{2}}$ \\
\hline$E$ & $E=D\left[\left(6 \alpha+\phi^{2}+\alpha^{2} \phi^{2}\right)(1-\rho)+8 \alpha \phi\left(\rho-\phi^{2}\right)\right] / 12 \alpha\left(1-\phi^{2}\right)$ \\
\hline$T$ & $T=E / v_{c}$ \\
\hline$e_{T}$ & $e_{T}=1-s\left[\phi^{2} D\left(1+\alpha^{2}\right)(1-\rho)+2 \alpha \phi D\left(\rho-\phi^{2}\right)-\alpha s\left(1-\phi^{2}\right) \rho\right] / \alpha \phi^{2}\left(1-\phi^{2}\right) D^{2}$ \\
\hline \multirow[t]{2}{*}{$O$} & $O=\left[H \Lambda s / 4 \alpha \phi\left(1-\phi^{2}\right) D\right] \max \left\{\alpha\left(1-\phi^{2}\right)+\left(2 \phi-\alpha^{2} \phi-\alpha^{3}\right)(1-\rho) / 2 ;\left[2\left(\phi-\alpha \phi+\alpha^{2}\right)\left(1-\alpha^{2}\right)+\alpha\left(\alpha^{2}-\phi^{2}\right)\right](1-\rho)\right\}$ \\
\hline & If $\alpha \leq \phi$ \\
\hline \multirow{4}{*}{$W$} & $W=\rho W_{c}+(1-\rho) W_{p}$ \\
\hline & $W_{c}=H \frac{D^{2}\left[\phi^{4}(8 \phi+3 \alpha)+\alpha^{3}\left(3 \alpha^{2}-2 \phi^{2}\right)-\alpha^{3}(\phi+\alpha)\right]-2 D s\left[\phi^{3}(2 \phi+\alpha)+\alpha^{3}(2 \alpha+\phi)\right]+6 \alpha^{3} s^{2}}{2}$ \\
\hline & $\begin{array}{c}12 \alpha \phi^{4} D^{2} \\
D\left[\phi^{3}(8 \phi+11 \alpha)+\phi^{2} \alpha(4+3 \alpha)-\alpha^{3}(\phi+\alpha)\right]-2 s\left[\phi^{2}(2 \phi+3 \alpha)+\alpha^{2}(3 \phi-2 \alpha)\right]\end{array}$ \\
\hline & $W_{p}=H C 12 \alpha \phi^{2}(\alpha+\phi) D$ \\
\hline$E$ & $E=D\left[\left(\alpha^{3}\left(2 \phi^{2}(1+\rho)-\alpha^{2} \rho\right)+\phi^{4}(12 \phi-7 \alpha)\right)(1+\phi)+6 \phi^{4}(1-\rho)\right] / 12 \phi^{4}(1+\phi)$ \\
\hline$T$ & $T=E / v_{c}$ \\
\hline$e_{T}$ & $e_{T}=1+\left[D\left(\phi^{2}-\alpha^{2}\right)+2 \alpha s\right]\left(\phi^{2}-\rho \alpha^{2}\right) / 2 \phi^{4} D-\left[2 \phi^{3} D-\rho \alpha^{2} s\right] s / \phi^{4} D^{2}$ \\
\hline$O$ & $O=\left(H \Lambda / 2 \alpha \phi^{2} D\right) \max \left\{\left[D \alpha\left(\phi^{2}-\alpha^{2}\right)\left(\phi^{2}-\rho \alpha^{2}\right)+4 s\left(\phi^{2} \alpha^{2}(1-\rho)+3\left(\phi^{4}-\rho \alpha^{4}\right)\right)\right] / 16 \phi^{2} ; s\left(\phi^{2}-\alpha^{2} \rho\right)\right\}$ \\
\hline
\end{tabular}

Tabla 2 - Formulación de los costes parciales de la estructura híbrida basada en transferencias

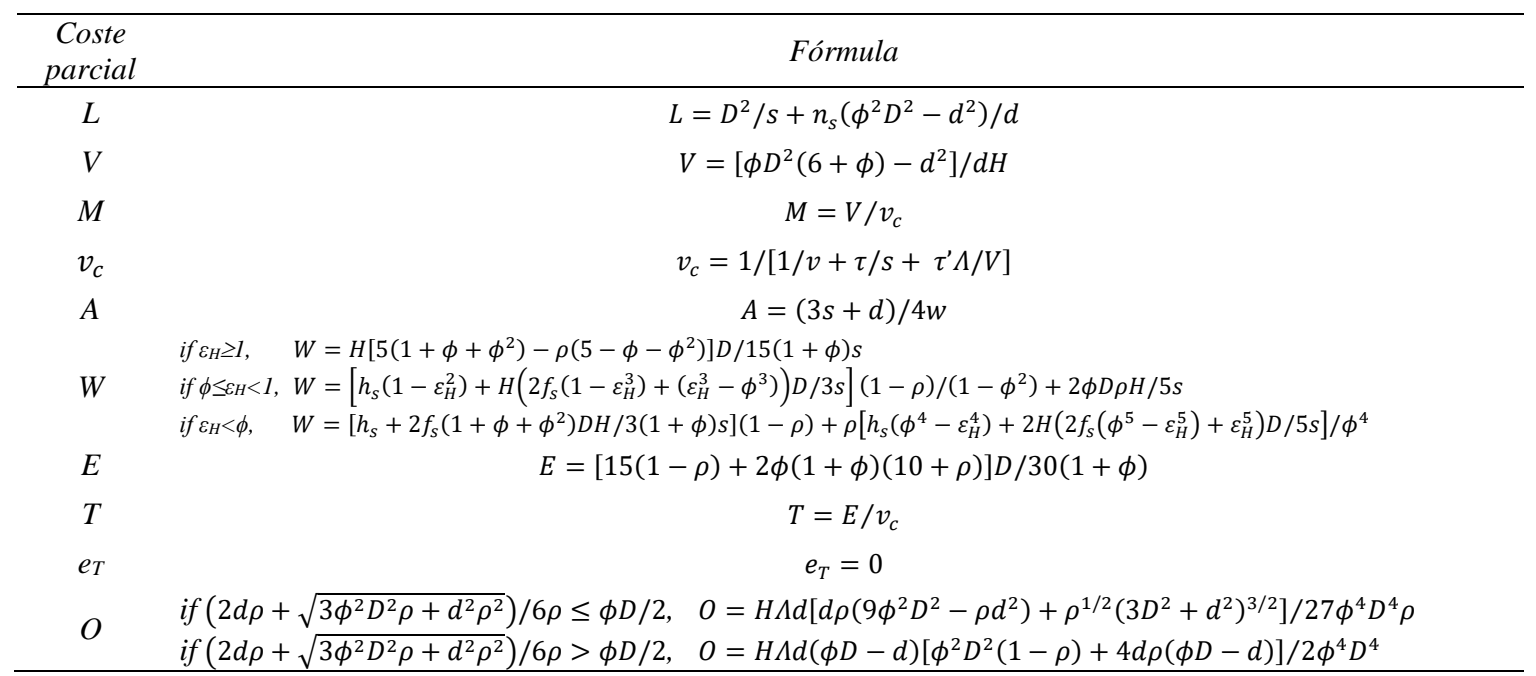

Tabla 3 - Formulación de los costes parciales de la estructura basada en servicios directos

\section{RESULTADOS DEL MODELO ANALÍTICO}

En este apartado, se presentan los resultados de aplicar el modelo analítico sobre un caso de estudio concreto. La Tabla 4 recoge los valores de los parámetros de entrada del modelo, que son los de una ciudad no específica, pero cuyo tamaño y demanda son similares a los de Barcelona. 


\begin{tabular}{|c|c|c|c|}
\hline Parámetro de entrada & Variable & Unidades & Valor \\
\hline Demanda en hora punta & $\Lambda$ & $\mathrm{pax} / \mathrm{hp}$ & 50,000 \\
\hline Demanda horaria media & $\lambda$ & $\mathrm{pax} / \mathrm{h}$ & 20,000 \\
\hline Tamaño de la ciudad & $D$ & $\mathrm{~km}$ & 7 \\
\hline Valor del tiempo & $\mu$ & $€ / \mathrm{h}$ & 15 \\
\hline Tiempo de transferencia & $\operatorname{tr}$ & $\min$ & 4 \\
\hline Velocidad de circulación & $v_{F F S}$ & $\mathrm{~km} / \mathrm{h}$ & 30 \\
\hline Velocidad peatonal & $w$ & $\mathrm{~km} / \mathrm{h}$ & 4.5 \\
\hline Coste unitario de infraestructura & $\epsilon_{L}$ & $€ / \mathrm{km}-\mathrm{h}$ & 76 \\
\hline Coste unitario de distancia recorrida & $\epsilon_{V}$ & $€ /$ veh-km & 0.85 o $1.1^{\mathrm{a}}$ \\
\hline Coste unitario por vehículo & $\epsilon_{M}$ & $€ /$ veh-h & 35 o $36^{\text {a }}$ \\
\hline Capacidad vehicular & $C$ & $\mathrm{pax} / \mathrm{veh}$ & 80 o $150^{\text {a }}$ \\
\hline Tiempo de parada & $\tau$ & $\mathrm{s}$ & 35 \\
\hline Tiempo de embarque por pasajero & $\tau^{\prime}$ & $\mathrm{s}$ & 3 \\
\hline Peso de percepción del tiempo de acceso & $w_{A}$ & - & 2.2 \\
\hline Peso de percepción del tiempo de espera & $w_{W}$ & - & 2.1 \\
\hline Peso de percepción del tiempo en vehículo & $w_{T}$ & - & 1.0 \\
\hline Peso de percepción del tiempo de transferencia & $w_{t r}$ & - & 2.5 \\
\hline Factor de seguridad de la capacidad vehicular & $S F$ & - & 1.2 \\
\hline Intervalo de corte entre tipos de servicio & $H_{s}$ & $\min$ & 12 \\
\hline Tiempo de espera de seguridad & $h_{s}$ & $\min$ & 5 \\
\hline Factor de coste de oportunidad & $f_{s}$ & - & $1 / 12$ \\
\hline
\end{tabular}

a El estudio considera dos tipos de flota: compuesta completamente por autobuses estándares o completamente por autobuses articulados.

\section{Tabla 4 - Parámetros de entrada del modelo para el caso de estudio}

La Figura 2 nos muestra la evolución de los costes totales del sistema con el proceso de descentralización de la demanda. En este caso se ha considerado que la densidad de demanda generada es igual en toda la ciudad, esto es, $f_{d}=1$, y como consecuencia $\rho=\phi^{2}$. De este modo con un único parámetro, en este caso $\phi$, definimos el grado de descentralización urbana.

Como se puede observar, la dispersión de la movilidad encarece el coste, pero de manera distinta para cada tipo de estructura. En etapas de alta centralización, por debajo de valores de $\phi$ de 0.1 , la red radial es la opción más adecuada. Sin embargo, cuando crece el grado de descentralización, los costes de esta red se disparan y es necesario un cambio de estructura. El desarrollo de una malla central presenta una evolución de costes similar en esas etapas iniciales, hecho que la invalida como estructura alternativa. Frente a esta opción, la red basada en conexiones directas presenta un encarecimiento menor, siendo el desarrollo de nuevas líneas que garanticen viajes directos la mejor opción.

Sin embargo, a partir de ciertos niveles de descentralización, la curva de costes de la red híbrida basada en transferencias reduce su pendiente, presentando un crecimiento menor que la otra alternativa. Esto deriva en la existencia de un punto de corte entre ambas, que nos indica el grado de descentralización $\phi_{V D-H}^{*}$ a partir del cual un cambio de estructura mejora la eficiencia de la red. En este caso de estudio, su valor está alrededor del 0.52. 


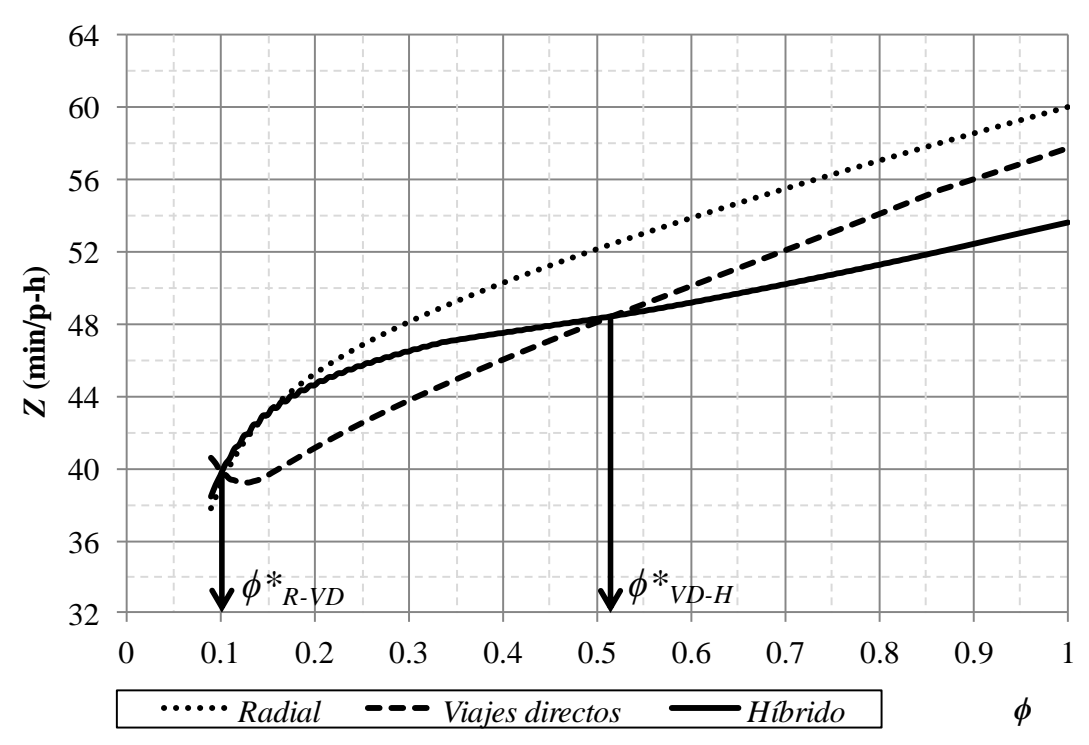

Fig. 2 - Evolución de los costes totales del sistema con el grado de descentralización de la demanda representado por el parámetro $\phi$

La red basada en conexiones directas reduce los costes del usuario ya que les evita transferir. Garantizar dicho objetivo es cada vez más costoso cuanta más extensa es el área atractora. Cada vez se necesita un mayor número de líneas y eso deriva en un elevado número de recursos dispersados entre todas ellas. Cuya consecuencia es en una red con bajo nivel de servicio para los coste que implica. Es por ello que un cambio en la estructura de red, reduciendo el número de líneas y concentrando todos los recursos en un número limitado de corredores, deriva en un sistema más eficiente. Esto supone un coste añadido para el usuario por tener que transferir, pero se compensa con la reducción de otros tiempos de la cadena de transporte.

En la Figura 3 se muestra como varía el valor de $\phi^{*}$ si se varía el tamaño de la ciudad y la demanda del sistema (siempre manteniendo la relación $\Lambda / \lambda=2.5$ ). Los resultados muestran que los niveles de dispersión que justifica el cambio de estructura, ya sea un cambio radialviajes directos o viajes directos-híbrida, es menor cuanto más grande es la ciudad.

La red radial presenta longitudes de viaje mayores, ya que un porcentaje muy elevado de los usuarios ha de pivotar en el centro de la ciudad para luego alcanzar su destino. Este aspecto se acrecienta en ciudades más extensas, lo que hace que pierda aplicabilidad esta estructura. Por otro lado, en relación a la red de viajes directos, para dar una adecuada cobertura espacial y temporal se necesitarían demasiadas líneas y recursos para menores niveles de dispersión, ya que al ser la ciudad más grande también lo es su área central. Por el contrario, la sinergia entre líneas a través de transferencias permite ofrecer un buen nivel de servicio con menos recursos en esos escenarios. 


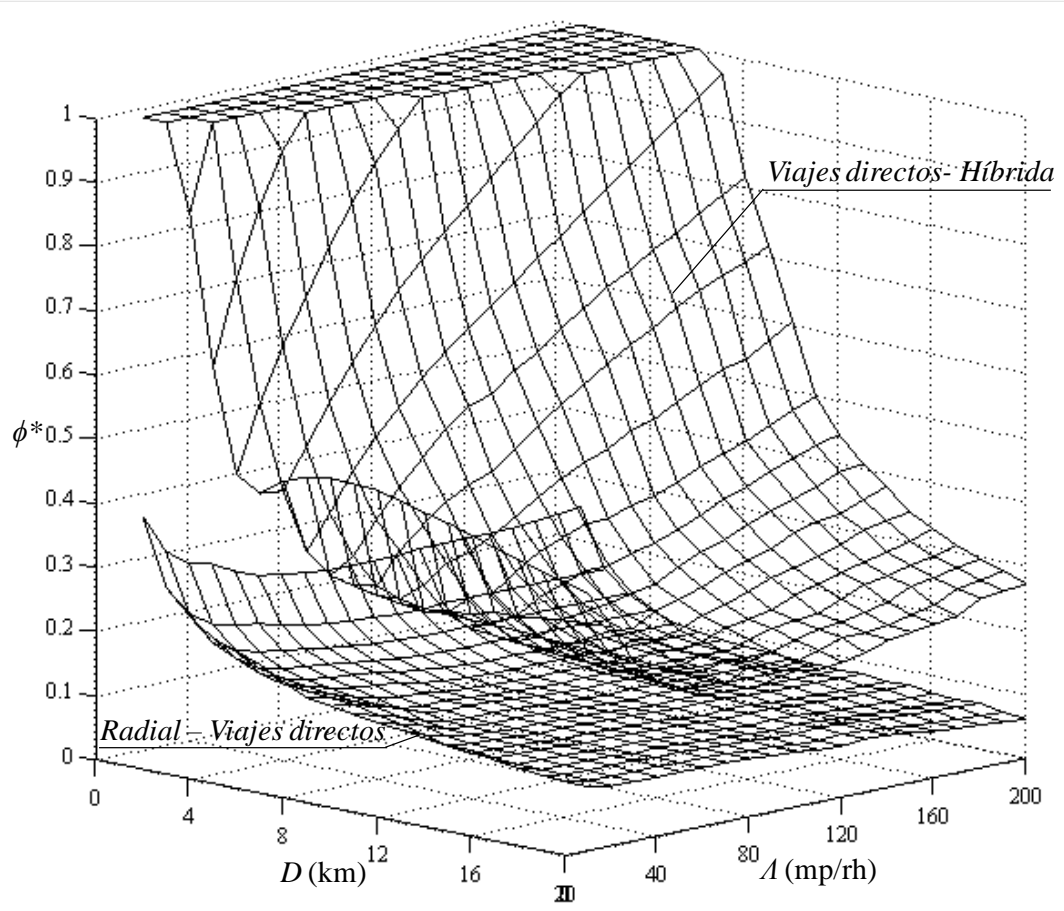

Fig. 3 - Grado de descentralización $\phi^{*}$ que justifica el cambio de estructura frente a la variación de la demanda de la red y el tamaño de la ciudad

En relación a la demanda, cuanto más elevada es, más se justifica la posibilidad de conexiones directas, en detrimento de las otras dos estructuras. Sin embargo, si la demanda es muy baja también gana aplicabilidad esta alternativa frente al esquema híbrido. El motivo es que cuando hay muy poca demanda la inversión es baja y al tener pocos recursos es mejor funcionar por horarios. En escenarios intermedios gana aplicabilidad la estructura basada en transferencias.

Las Figuras 4 y 5 muestran las áreas de aplicabilidad de cada una de las estructuras en función del coste por transferencia y del parámetro $f_{d}$ respectivamente. El primero de ellos es un factor clave, ya que la transferencia es el principal elemento que distingue el modo en que operan las estructuras comparadas. Esto lo confirman los resultados, donde en algunos escenarios la red de viajes directos y en otros la red híbrida dejan de tener aplicabilidad.

Por lo que respecta al segundo parámetro, el hecho de que la demanda generada presente mayor o menor concentración no produce grandes cambios en la aplicabilidad de las estructuras. Los costes totales del sistema se reducen, pero de manera similar en todas ellas, lo que deriva en variaciones acotadas del parámetro $\phi^{*}$. El parámetro $\phi$ condiciona en mayor medida que estructura de red es la óptima en un escenario frente al parámetro $f_{d}$. Es decir, el tamaño del área central atractora frente a como se distribuye la generación de la demanda. 


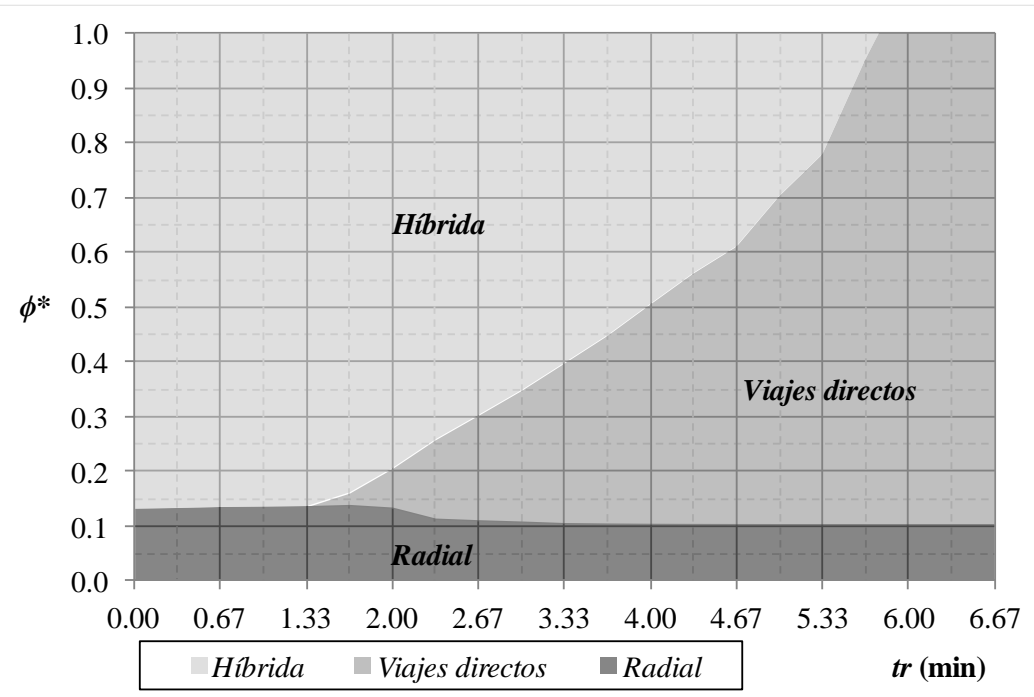

Fig. 4 - Grado de descentralización $\phi^{*}$ que justifica el cambio de estructura frente a la variación del coste de transferencia

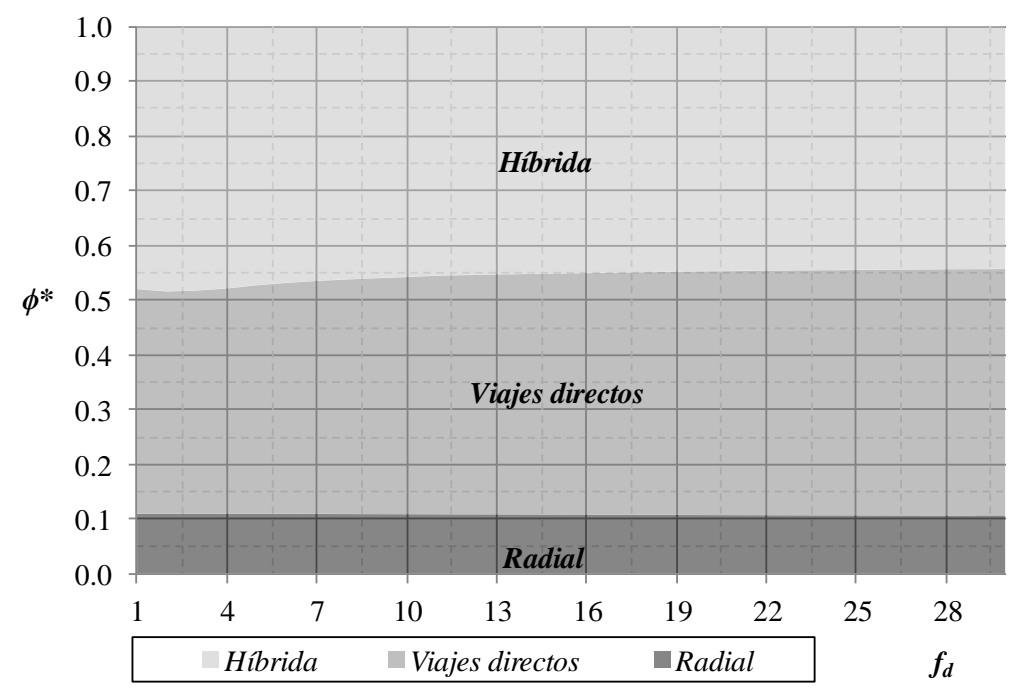

Fig. 5 - Grado de descentralización $\phi^{*}$ que justifica el cambio de estructura frente a la variación del factor de densidades $f_{d}$

\section{DISPERSIÓN DE LA MOVILIDAD EN CIUDADES REALES}

En este apartado se busca trasladar a ciudades reales los resultados analíticos previos. Es decir, utilizar dichos resultados para discernir en qué situación se encuentra una ciudad, y por tanto, que estructura de red debe ser utilizada. En primer lugar se debe determinar cómo medir esa descentralización. Galster et al (2001) presentaba hasta ocho dimensiones que indican el grado de dispersión de una ciudad: densidad, continuidad, concentración, clusterización, centralidad, nuclearidad, usos mixtos y proximidad. Sin embargo, unas y otras guardan dependencia, por ello, el análisis de algunas de ellas ya nos puede indicar la evolución de las demás.

Otros autores hacen un análisis más simplificado del grado de dispersión. Lee (2007) lo hace 
a través de índices de centralización y concentración. Los primeros indican el grado en que las actividades están localizadas alrededor del centro tradicional, mientras que los segundos informan si dichas actividades se localizan en pocos o muchos emplazamientos. Respecto a la centralización se utilizan índices como el de Wheaton o la distancia media ponderada al centro, y para la concentración índices de inequidad como el coeficiente de Gini. Tsai (2005) combinaba el grado de distribución mediante el coeficiente de Gini, y el grado de clusterización a través de coeficientes de econometría espacial como los índices de Moran o Geary, permitiendo conocer si la ciudad presenta más de un centro. Pereira et al (2013) propuso un índice de centralidad urbana producto de la combinación de un índice de distribución y un índice de proximidad de las actividades.

En base a los estudios previos, en este artículo se trabaja con dos de las anteriores dimensiones: la inequidad en la distribución de las actividades sobre la ciudad y la estructura de esta, si es monocéntrica o policéntrica. El modelo analítico trabaja con una estructura monocéntrica, la cual presenta un mayor o menor grado de descentralización. Conocer la estructura urbana es el punto de partida del análisis. Se debe confirmar que la ciudad presenta una estructura predominantemente monocéntrica. Esto es determinado a través del índice de Moran (Anselin, 1995), que indica la existencia de autocorrelación espacial de una variable y a su vez donde se concentran los clústers de alta y baja demanda de dicha variable.

Por otro lado, el parámetro $\phi$ marca si la demanda se distribuye sobre una pequeña o extensa área en torno al centro tradicional. Un índice de inequidad como el coeficiente de Gini es una medida comparable con el parámetro del modelo una vez se haya determinado la estructura monocéntrica de la ciudad.

\subsection{Análisis de la matriz origen-destino de la ciudad de Barcelona}

Una fuente de información que permite tener una idea del grado de dispersión de la movilidad son las matrices origen-destino. Estas indican la capacidad de generación y atracción de viajes de las diferentes zonas de transporte en que se divide la ciudad. El análisis se hace en dos niveles. Primero un análisis de la estructura de la ciudad que informa sobre si esta es monocéntrica, y después, se estima el coeficiente de Gini para conocer el grado de concentración de la demanda.

El caso de estudio que aquí se expone es el de la ciudad de Barcelona. Donde el análisis econométrico de su matriz de viajes muestra que presenta una estructura monocéntrica. El índice de Moran global, cuyo valor es 0.59 , nos indica que existe autocorrelación de los valores de densidad de demanda entre las diferentes zonas de transporte. Es decir, las zonas de alta densidad de demanda se localizan unas cercanas a las otras, igual que hacen entre si las de baja densidad. El análisis local lo confirma como muestra la Figura 4b, donde se observa la existencia de un clúster de alta demanda (zonas de color rojo) en torno al centro tradicional de la ciudad, y clústers de baja demanda (zonas de color azul) conformados por 
las zonas más periféricas. De este modo, se confirma que en Barcelona el patrón de movilidad es predominantemente monocéntrico.

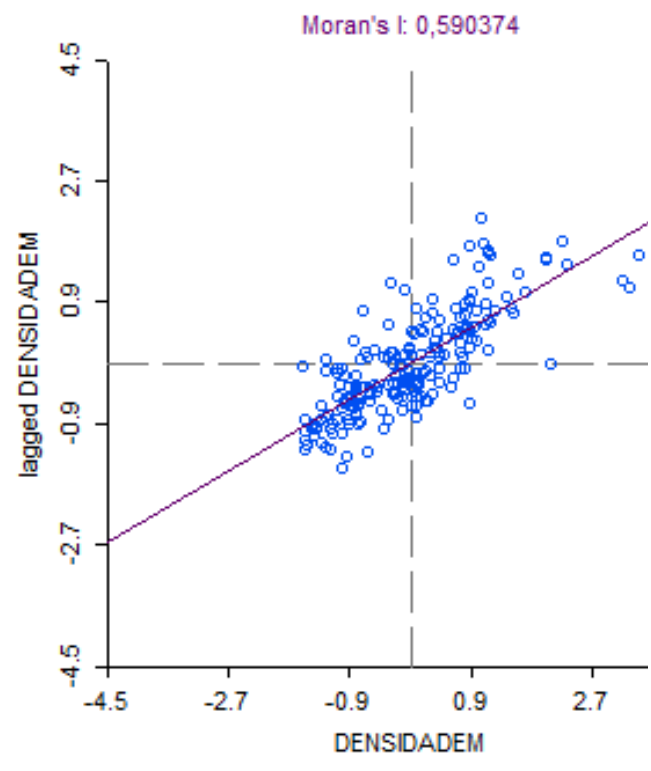

(a)Diagrama de dispersión de Moran

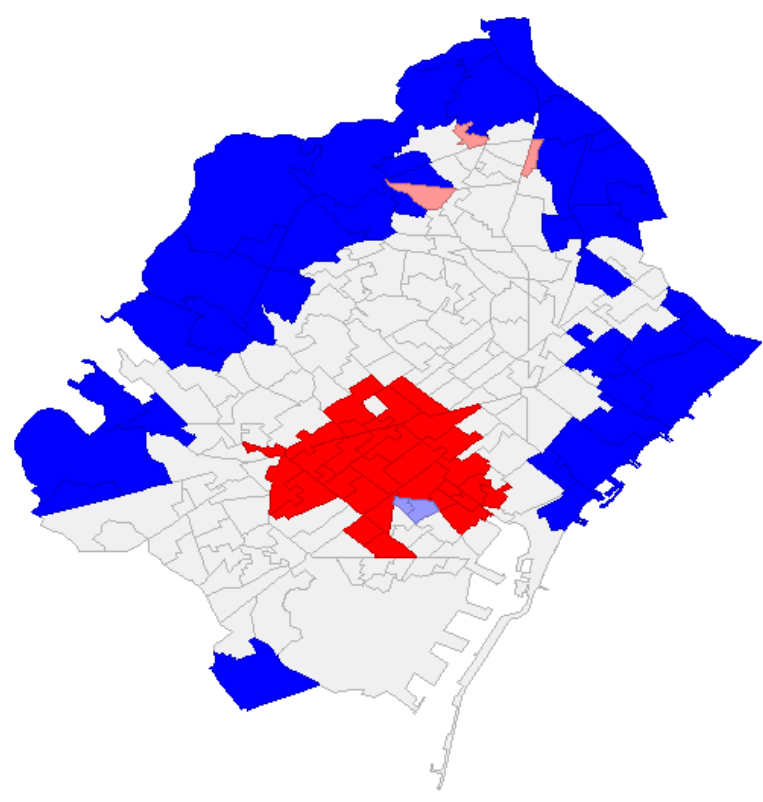

(b)Mapa de clústers de demanda

Fig. 4 - Diagrama de dispersión de Moran y mapa de clústers de alta y baja demanda

A continuación, en la Figura 5 se muestra la curva de Lorenz para Barcelona (curvas continuas), donde se distinguen las curvas de demanda generada y atraída. La segunda presenta un mayor grado de concentración. La ciudad presenta un índice de Gini de valor 0.50 para la demanda generada y de valor 0.57 para la atraída. La curva muestra tres tipos de zonas en función de su densidad de demanda. Un $40 \%$ de la ciudad que presenta valores bajos, un $30 \%$ con una densidad intermedia, y un $30 \%$ con una densidad alta. Del análisis anterior, se puede deducir que a rasgos generales cada una de estas categorías tienen una localización asociada: periferia, espacio intermedio y entorno al centro tradicional respectivamente.

El modelo analítico simplifica la distribución de la demanda. Esto conforma unas curvas de Lorenz como las que se muestran en la Figura 5 (curvas discontinuas). La curvas tiene asociado el mismo coeficiente de Gini que la curva real de Barcelona correspondiente. El área central del modelo representaría un área que en Barcelona acumula algo más del $80 \%$ de los viajes.

El coeficiente de Gini $(C G)$ y el parámetro $\phi$ siguen la relación $\phi^{2}=1-C G$. Por lo tanto, el parámetro $\phi$ en el caso de Barcelona es 0.66. La relación de densidades de demanda generada es $f_{d}=18.47$. Si localizamos estos valores en la Figura 5 , se puede determinar en qué escenario se encuentra la ciudad de Barcelona. En ella, una estructura basada en transferencias sería más eficiente, ya que su $\phi$ es superior a 0.55 , es decir, la ciudad se encuentra en el área de aplicabilidad de la red híbrida. 


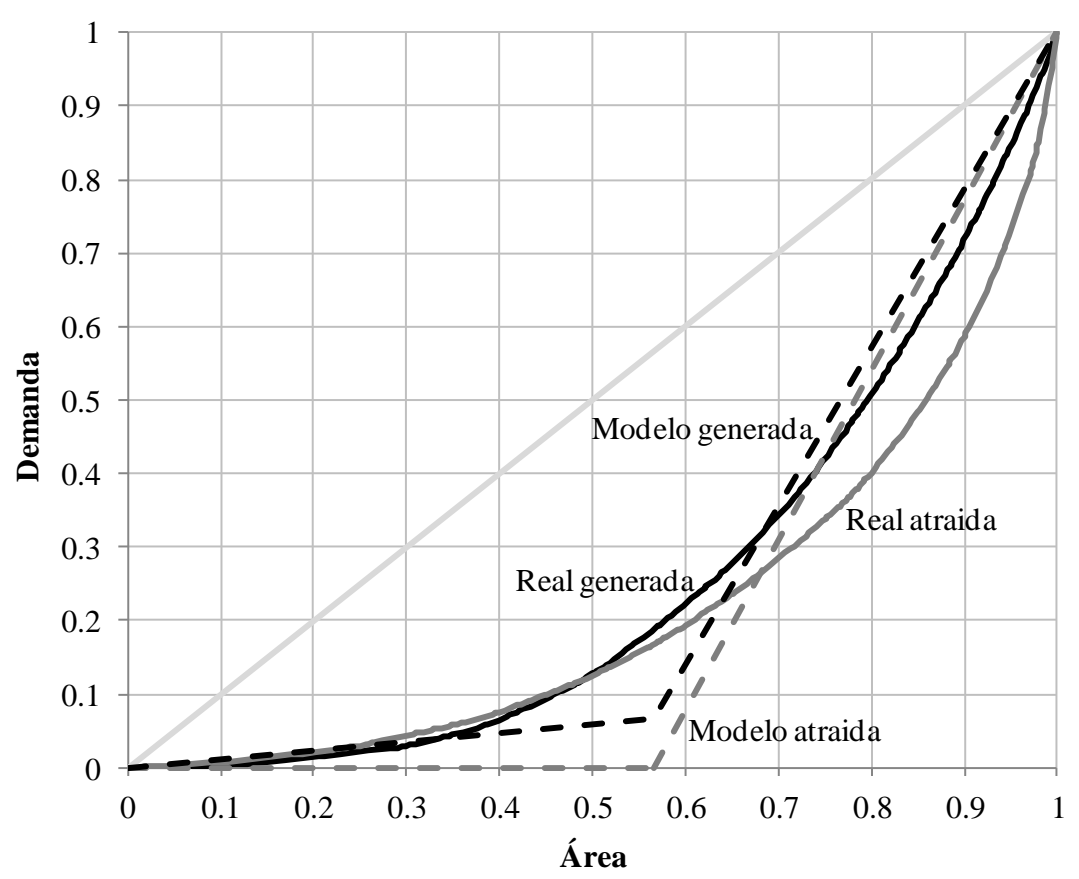

Fig. 5 - Curvas de Lorenz de la demanda generada y atraída de Barcelona, y las curvas de mismo coeficiente de Gini para el caso del modelo analítico

\section{CONCLUSIONES}

La discusión sobre que estructura de red es la más adecuada no tiene una respuesta única, depende del grado de dispersión en la que se encuentre la ciudad de estudio. La red radial es adecuada para elevados grados de centralización urbana, pero pronto deja de ser competitiva. La estructura basada en conexiones directas es más competitiva si la demanda está concentrada en un espacio reducido. Sin embargo, deja de serlo cuando esta se dispersa, ya que demasiadas líneas se necesitarían para garantizar esos viajes directos. Es entonces cuando un cambio de estructura basado en transferencias es la mejor alternativa para alcanzar un servicio lo más eficiente posible.

Lo que el modelo también muestra es que ese grado de dispersión a partir del cual el cambio de estructura resulta la mejor decisión posible es variable. Este depende de las características de la ciudad, la demanda o la penalización que el usuario percibe por tener que transferir. Ciudades grandes con niveles de demanda razonables son proclives a encontrarse en un escenario donde una estructura basada en transferencias está justificada. Por supuesto, para el éxito de este tipo de redes, un adecuado diseño de los puntos de transferencia es esencial.

En todo caso, el modelo puede ser una herramienta útil para conocer en que escenario se encuentran las ciudades hoy en día. El caso estudiado es el de la ciudad de Barcelona, donde su grado de dispersión está por encima del nivel de descentralización que justifica una estructura híbrida. Para futuros trabajos, el estudio de otras matrices origen-destino ayudará a conocer de manera más generalizada la situación actual de los entornos urbanos y sacar conclusiones en que tipos de ciudades es más adecuado optar por cada tipo de estructura. 


\section{AGRADECIMIENTOS}

El autor del artículo ha sido financiado a través de una beca para la Formación de profesorado universitario (FPU), cuyo número de referencia es AP2010-4544, del Ministerio de Educación del Gobierno de España.

\section{REFERENCIAS}

Anselin, L. (1995). Local indicators of spatial association - LISA. Geographical Analysis 27 (2), pp. 93-115.

Badia, H., Estrada, M., Robusté, F. (2014). Competitive transit network design in cities with radial street patterns. Transportation Research Part B 59, pp. 161-181

Badia, H., Estrada, M., Robusté, F. (2016). Bus network structure and mobility pattern: A monocentric analytical approach on a grid street layout. Working paper.

Daganzo, C.F. (2010). Structure of competitive transit networks. Transportation Research Part B 44 (4), pp. 434-446.

Estrada, M., Roca-Riu, M., Badia, H., Robusté, F., Daganzo, C.F. (2011). Design and implementation of efficient transit networks: Procedure, case study and validity test. Transportation Research Part A 45 (9), pp. 935-950.

Jara-Díaz, S. R., Gschwender, A. (2003). From the Single Line Model to the Spatial Structure of Transit Services: Corridors or Direct?. Journal of Transport Economics and Policy 37(2), pp. 261-277.

Mees, P. (2000). A very public solution: transport in the dispersed city. Melbourne University Press, Melbourne.

Thompson, G.L. (1977). Planning considerations for alternative transit route structures. Journal of American Institute of Planners 43 (2), pp. 158-168.

Thompson, G.L., Matoff, T.G. (2003). Keeping up with Joneses: planning for transit in decentralizing regions. Journal of the American Planning Association 69 (3), pp. 296-312.

Tirachini, A., Hensher, D.A., Jara-Díaz, S.R. (2010). Comparing operator and users costs of light rail, heavy rail and bus rapid transit over a radial public transport network. Research in Transportation Economics 29 (1), pp. 231-242.

Vaughan, R. (1987). Urban spatial traffic pattern. Pion Ltd., London.

Zhao, F. (2006). Large-scale transit network optimization by minimizing user cost and transfers. Journal of Public Transportation 9 (2), pp. 107-129 